\title{
Current pain management strategies for patients with erythromelalgia: a critical review
}

This article was published in the following Dove Press journal: Journal of Pain Research

\section{See Wan Tham ${ }^{1,2}$ \\ Marian Giles ${ }^{1,2}$}

'Seattle Children's Research Institute, Seattle, WA, USA; ${ }^{2}$ Department of Anesthesiology and Pain Medicine, University of Washington School of Medicine, Seattle, WA, USA
Correspondence: See Wan Tham Seattle Children's Research Institute, CW8-6 PO Box 537I, Seattle,

WA 98।45-5005, USA

$\mathrm{Tel}+\mathrm{I} 206884$ I278

Fax + I 2069853262

Email see.tham@seattlechildrens.org

\begin{abstract}
Erythromelalgia (EM) is a rare disorder characterized by erythematous, warm, painful extremities, which is often precipitated by cold conditions. The pathophysiology of EM is incompletely understood. Recent investigations have identified sodium channelopathy as a genetic cause for this pain condition, classified as primary inherited EM. Other subtypes are idiopathic EM and secondary EM. The management of pain in EM is challenging as no single therapy has been found to be effective. There is varying response to pharmacotherapy and significant variability within this clinical population, resulting in a stepwise trial and error approach. Consequently, EM is often associated with poorer health-related quality of life with higher morbidity. There is currently no consensus or guidelines on management of pain in EM. This is a review of the literature on management of pain using pharmacologic, procedural intervention and nonpharmacologic treatment in children and adults with EM.
\end{abstract}

Keywords: erythromelalgia, pain, voltage gated sodium channel, therapy

\section{Introduction}

Erythromelalgia (EM), previously also known as erythermalgia, was initially described in 1878 by Mitchell, with hallmark symptoms of red (erythros) limbs (melos) that are painful (algos). ${ }^{1}$ This was subsequently subdivided into primary, idiopathic, and secondary forms in 1938 by Smith and Allen, reflecting our understanding of the polymorphic etiologies that may contribute to this disorder. ${ }^{2}$ EM is a challenging condition to manage. There are several factors that have limited a systematic approach to management of the disease. First, the underlying pathophysiology remains poorly understood, thereby limiting intentional treatment of pain. Second, EM is a rare disorder, estimated to affect 0.36-2 per 100,000 people per year in the USA and Europe, ${ }^{3-5}$ making it a difficult subject of research. Finally, the trajectory of symptoms and the long-term prognosis for patients are not well understood. Although there are promising studies that may provide clarity for the future interventions, therapies currently available remain unfortunately ineffective for many patients.

The classic presentation of EM is the triad of severe paroxysmal burning pain, erythema, and increased skin temperatures in the extremities. ${ }^{6,7}$ There are isolated reports of facial, auricular and genital involvement. ${ }^{8-10}$ EM can present at any age with onset commonly reported in the first decade of life, although it is most often diagnosed in the fifth and sixth decades. ${ }^{11}$ Pain is exacerbated by increased ambient temperatures, exercise, and limb dependence. Relief is often achieved using cooling strategies such as immersion of limbs in ice-cold water, application of cold socks/ 
gloves, and decreasing ambient heat (eg, ventilation, fans). Profound complications from chronic cold exposure have been reported, including maceration, ulceration, windburn, hypothermia, and frostbite. ${ }^{12}$ Infections, sepsis, and amputation have been linked to cold exposure. ${ }^{13}$ The course of EM is variable, with exacerbations and remissions. ${ }^{14,15}$ Of critical impact is the persistent and severe nature of pain that has been found to impair physical, psychological, and social functioning, resulting in poorer quality of life, with higher rates of morbidity and mortality. ${ }^{16}$

The etiology of EM is incompletely understood. Genetic research has identified a link between cases of inherited EM and mutations in the Nav1.7 sodium channel encoded by the $S C N 9 A$ gene. ${ }^{17,18}$ Nav1.7 subtypes are preferentially expressed in sensory and sympathetic ganglia neurons, and mutations result in neuronal excitability by reducing current threshold and enhancing repetitive firing in dorsal root ganglia. ${ }^{19,20}$ These mutations have rarely been detected in sporadic or secondary cases. Patients with SCN9A mutations have been classified as primary inherited EM. The other category of primary EM has been denoted as idiopathic, describing sporadic cases where a specific genetic mutation has not or cannot be identified.

Neuropathological and microvascular functional changes are implicated the pathophysiology of the secondary forms of EM. Studies have identified neurogenic dysregulation of cutaneous sympathetic fibers and impaired function as a potentially causative finding in patients with EM. ${ }^{21}$ Investigations such as thermoregulatory sweat testing and quantitative sudomotor axon reflex test supported the diagnosis of small fiber neuropathy in patients with EM. ${ }^{22-24}$ Other studies suggest vascular causes, with pain from chronic perfusion deficit and tissue ischemia..$^{25,26}$

To date, there is no therapy that has demonstrated consistently high efficacy across individuals with EM, highlighted by the lack of consensus or guidelines on the treatment of EM. The aim of this study was to conduct a systematic review of pain treatment for EM and provide an update on treatment options. The published data are gathered from small case series, retrospective cohorts, case studies, and several randomized controlled trials (RCTs).

\section{Methods}

A literature search was performed using PubMed, Ovid, and Web of Science databases, yielding an initial total of 2,693 records. The search term was "erythromelalgia" in the title and as the MeSH term. The inclusion criteria were studies on EM, published after 1985 and in English.
After excluding duplicates ( $\mathrm{n}=801$ ), we identified 1,892 citations using the search criteria. Of the 1,892 citations, we excluded 1,177 that did not fulfill the inclusion criteria. From a review of the abstracts of the remaining 715 studies by SWT and MG, 296 were found eligible for inclusion based on the information provided in the abstract for treatment of pain in EM. Reasons for excluded citations were studies that were not relevant, other diagnosis not related to EM, and basic science reports. Of these 296 studies, full-text reports were assessed for eligibility, giving a final number of 292 relevant studies (Figure 1). There were 5 RCTs, 1 non-RCT, 6 retrospective studies, 3 studies on nonpharmacologic management, 13 observational studies on EM, 29 case series, 178 case reports, and 57 review articles.

\section{Results}

This review will provide details on the pharmacologic, nonpharmacologic, and procedural interventions for pain management. Areas of intervention include trigger management, treatment of underlying pathophysiology, and symptomatic management of pain. The majority of the evidence was gathered from small case series studies, retrospective cohorts, or case reports.

\section{Trigger management}

Patients with EM often employ strategies to avoid situations that may precipitate pain (typically induced by heat) and the use of cold exposure reduction. Avoidance of heat includes being in cold ambient environments (air conditioning), limiting physical activities, avoiding footwear, and moving to colder environments. Patients repeatedly immerse their limbs in cold water and apply ice to the extremities. It is important to caution patients that impaired skin integrity and microcirculatory abnormalities from overexposure to water and cold can result in ulceration, maceration, and infections. ${ }^{12,27}$ Furthermore, there is a concern that patterns of increasing pain in EM may, in fact, be associated with the overuse of cooling strategies. ${ }^{12}$ Therefore, patients need to be counseled on the appropriate use of cooling strategies, balancing tolerance of pain, and functional activities.

\section{Pharmacotherapy}

Many pharmacologic agents have been used to treat EM over the decades. Vascular agents (aspirin, prostaglandins), sodium channel blockers (lidocaine, mexiletine, carbamazepine, oxcarbazepine), antidepressants (tricyclic antidepressants, serotonin reuptake inhibitors), anticonvulsants, 


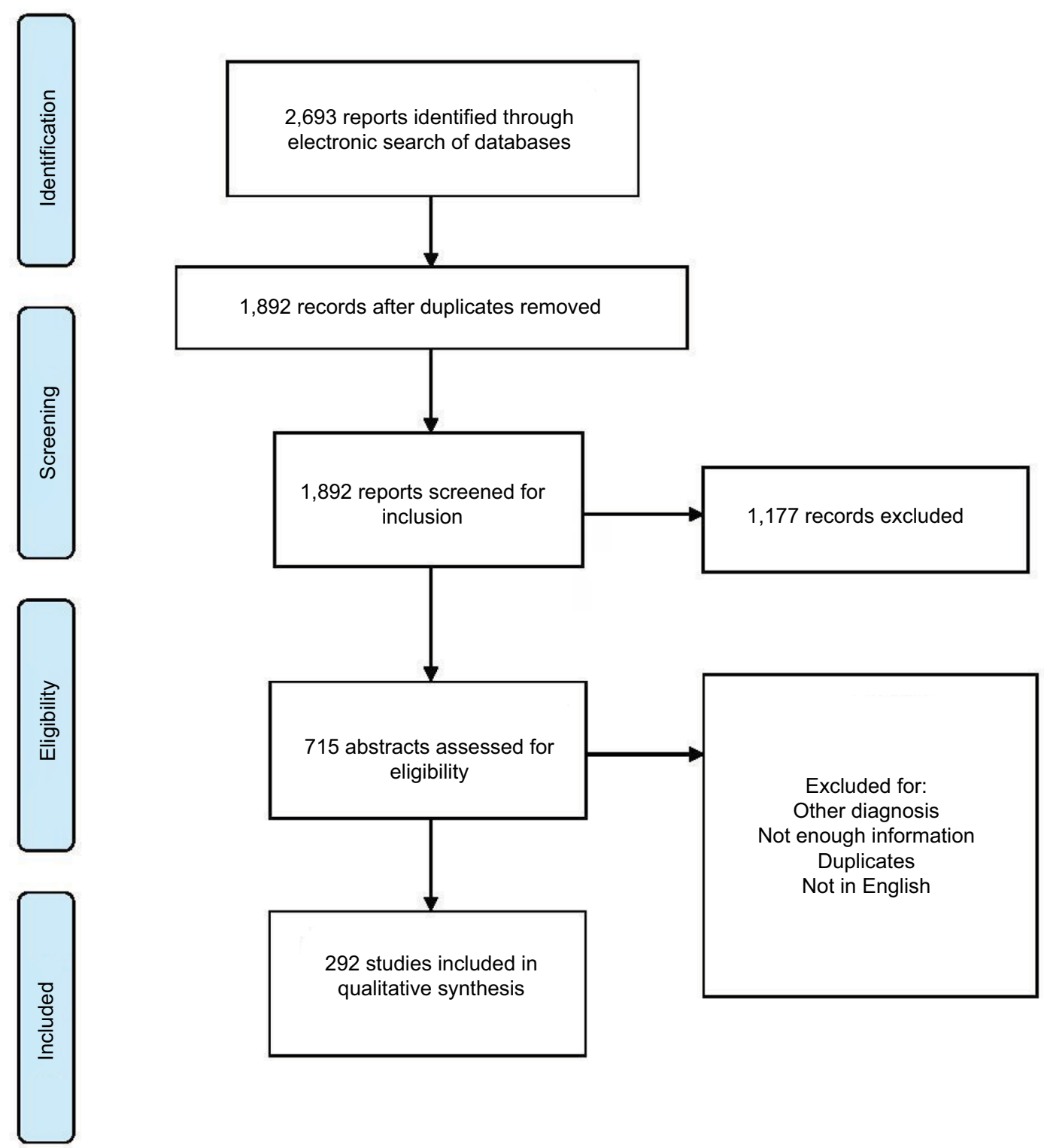

Figure I Flowchart of literature search.

antihistamines, topical application of medications, and immunosuppressant drugs have been trialed with no single agent demonstrating complete efficacy, thereby resulting in polypharmacy for the majority of patients. These agents have been administered with the goal of targeting underlying etiology and/or for symptom management.

\section{Aspirin/ticlopidine}

Aspirin has been considered as a first-line treatment, likely due to early studies reporting its efficacy and the low side effect profile. There is more evidence that aspirin is effective for patients with associated thrombocythemia and polycythemia vera. ${ }^{28,29}$ It is hypothesized that this is secondary to its antiplatelet action. Caution should be exercised due to potential gastrointestinal side effects (eg, hemorrhages) of aspirin at high doses. Ticlopidine is an alternative agent that has been trialed and found to be better tolerated. ${ }^{30}$

\section{Nonsteroidal anti-inflammatory agents}

There appears to be a limited role for nonsteroidal antiinflammatory drugs other than aspirin in the treatment of EM. Piroxicam has been documented as effective in cases of aspirin allergy, ${ }^{31}$ and indomethacin has been used for cases of facial or auricular EM due to its ability to penetrate the blood brain barrier and its potential role in reducing facial parasympathetic outflow. ${ }^{32}$ 


\section{Prostaglandins}

Sympathetic dysfunction, arteriovenous shunting of the skin, insufficient perfusion, and tissue hypoxia have been proposed to contribute to pain in EM. ${ }^{33}$ Prostaglandin and prostacyclin analogs with vasodilatory effects have been the subjects of two small RCTs in EM treatment of pain $(n=12-21)$. Findings were that iloprost and misoprostol were associated with a reduction in symptoms and sympathetic dysfunction. ${ }^{7,34,35}$

\section{Sodium channel blockers}

Drugs that target the underlying genetic cause of cases of hereditary EM (Nav1.7 sodium channel) have proved promising in the preclinical setting. Two proof-of-concept RCTs showed that agents developed specifically to antagonize the abnormal Nav1.7 sodium channel improved symptoms and neurophysiologic markers in patients with an identified SCN9A mutation. ${ }^{36,37}$ However, these agents are yet to be available in clinical practice.

Lidocaine and mexiletine provide nonselective partial blockade of voltage-gated sodium channels, working on segment 6 of domain IV of the alpha subunit to shorten the action potential duration. Lidocaine is an amide-type local anesthetic agent that stabilizes neuronal membranes by inhibiting the ionic fluxes required for the initiation and conduction of impulses. Lidocaine has been used in the treatment of EM on a trial basis. ${ }^{38-40}$ However, lidocaine has a narrow therapeutic window with significant cardiac, cognitive, and motor side effects. Further research has also identified that different genetic EM mutations may be responsible for the varying responses of individuals with hereditary EM to lidocaine treatment. Therefore, identifying the specific SCN9A genotype may provide guidance on treatment. ${ }^{41,42}$ Due to limited availability of genetic testing, current approach to the use of lidocaine may involve a trial and error strategy. ${ }^{43}$

Positive response to lidocaine infusions has been considered as an indicator of success with mexiletine, an orally available form of lidocaine. Mexiletine has been documented to reduce pain episodes in case reports of children and adults. ${ }^{44-47}$ However, similar to lidocaine, there is variable clinical response that may be also explained by the genetic polymorphisms in Nav1.7 mutations, some of which may have a stronger response to lidocaine and mexiletine than others. ${ }^{48}$

Carbamazepine and oxcarbazepine are also often utilized as first-line agents due to their mechanism of action on inactivation of sodium channel subtypes, including Nav1.7. They reduce the excitability of sensory and sympathetic neurons.
Carbamazepine has not been found to have significant effects on pain in EM. However, there is some evidence suggesting that individuals who carry a specific mutation on the Nav1.7 sodium channel (V400M) may be more responsive to carbamezepine. ${ }^{4-51}$ Oxcarbazepine has been used in inherited EM. It has been found to be better tolerated by patients compared to carbamazepine. ${ }^{49}$

\section{Calcium channel blockers}

Calcium channel antagonists have been proposed for use to attenuate the vasoconstriction and reactive hyperemia in EM. Amlodipine, nifedipine, and diltiazem have been utilized with isolated reports of remission. ${ }^{52}$ Use of high-dose oral magnesium has been reported in 12 patients recruited via an informal survey through The Erythromelalgia Association. Of these patients, $61.5 \%$ reported varying degrees of improvement. Two patients' dose titration was limited by diarrhea as an adverse effect. ${ }^{52}$ In addition to calcium channel antagonism, magnesium was proposed to have sympatholytic and muscle relaxant qualities to account for potential efficacy.

\section{Antidepressants}

Various classes of antidepressants have been used. Selective serotonin and norepinephrine reuptake inhibitors such as fluoxetine, sertraline, and venlafaxine have also been used with reported remission. ${ }^{53,54}$ Serotonin antagonists such as methysergide and pizotifen have been tried with some reported effectiveness. ${ }^{110,111}$ These agents may affect vascular control by inhibition of serotonin reuptake and act on sympathetic fibers by inhibition of reuptake of noradrenaline. Data on tricyclic antidepressants (eg, amitriptyline, imipramine; SNRI, eg, duloxetine) are limited to case reports or series, often with variable responses and in conjunction with polypharmacy. ${ }^{55}$

\section{Anticonvulsants}

Gabapentinoids such as gabapentin and pregabalin are commonly used to treat neuropathic pain. This has been extended for potential application in the neuropathic nature of pain described in EM. The hypothesized analgesic action involves the voltage-gated L-type calcium channels. Gabapentin has a well-established safety profile with the most common adverse effect being sedation, which may be self-limiting. Gabapentin has been used with variable success in the treatment of EM, often combined with other medications. ${ }^{14,49,56}$ Pregabalin is a structural analog of gamma-aminobutyric acid, similar to gabapentin. It binds to the alpha 2 delta ligand of the voltagegated calcium channels in the central nervous system that are proposed to exert its nociceptive and anticonvulsant activity. 
It appears to act as a presynaptic inhibitor of the release of excitatory neurotransmitters including glutamate, substance $\mathrm{P}$, and calcitonin gene-related peptide. Common side effects are dizziness, somnolence, dry mouth, and weight gain. The case reports show that this has been used in EM with limited success. ${ }^{57,58}$ Both agents are started at a low dose and titrated to effect.

\section{Antihistamines}

Antihistamines may have potent vascular effects and may be considered for treatment. Cyproheptadine and pizotifen are antihistamines with serotonin antagonist effects at 5-HT2 receptors. ${ }^{59}$ A survey of the members of The Erythromelalgia Association reported marked improvement in $40 \%$ of patients with antihistamine, but not in $60 \% .{ }^{55}$ This includes use of desloratadine, chlorpheniramine, and diphenhydramine. In contrast, non-sedating antihistamine cetirizine hydrochloride did not show improvement, except in a pediatric patient as shown by a case report. ${ }^{60}$ It has been proposed that there may also be longstanding local allergic reaction in EM, for which antihistamines may have some efficacy.

\section{Sodium nitroprusside}

Sodium nitroprusside has been used to treat EM associated with hypertension in both adults and children. ${ }^{61,62}$ Hypertension has been reported in a large number of patients with EM. The proposed mechanism of the analgesic effect for sodium nitroprusside may be related to its mechanism of arteriolar vasodilation.

\section{Topical agents}

As the symptoms of EM appear to be localized, the topical route may be preferable to reduce the systemic effects of medications. Different compounds have been used, including topical amitriptyline-ketamine, lidocaine, capsaicin, and midodrine. In a retrospective case series of 32 adults prescribed amitriptyline-ketamine topical compound, over $70 \%$ had some or substantial relief of pain, with no adverse effects. ${ }^{63}$ The penetration of topical lidocaine into intact skin was proposed to be sufficient to produce analgesic effect. ${ }^{64,65}$ Topical midodrine $0.2 \%$, an alpha 1 agonist, was used for its vasoconstriction properties and was found to be successful in symptom reduction for 12 patients. ${ }^{66} \mathrm{~A}$ single case report of capsaicin cream was identified, with reported success. ${ }^{67}$

\section{Immunosuppressant agents}

There are a number of case reports and series on the use of immunosuppressants. One theory is that an autoimmune etiology and small fiber neuropathy may underlie EM. There are ongoing investigations to further determine whether there may be a subgroup classification of corticosteroid-responsive patients with EM, particularly in whom there were documented antecedent infections, trauma, or surgery as potential precipitants. ${ }^{68}$ Several case reports have documented clinical recovery for patients with comorbid diagnoses of both small and large fiber neuropathy after treatment with intravenous immunoglobulin infusions, methylprednisolone, and oral corticosteroids. ${ }^{43,69-71}$ The role of immunosuppressants in the treatment of EM remains unclear, with limited data and lack of clarity regarding the role of immune function in the development of EM.

\section{Beta-blockers}

Beta blockers have been used for EM, specifically to treat vulvar involvement. ${ }^{8}$ Specific mechanisms were not proposed, except that nebivolol may have vasodilatory effects.

\section{Nicotinic acid}

Nicotinic acid has been used for mushroom poisoning; Paralepistopsis acromelalga, formerly known as Clitocybe acromelalga, is a rare poisonous mushroom. The mycotoxins in this mushroom cause symptoms resembling those of EM; however, its pathogenesis remains unclear. ${ }^{72}$

\section{Opioids}

None of the case reports reviewed had reported opioids to be useful as a sole therapy. On the contrary, many studies report that opioids, even in combination with other medications, are not helpful in controlling the severe pain found in EM patients. ${ }^{73}$ As there are risks (tolerance, sedation, respiratory depression, constipation) associated with chronic opioid use, caution must be exercised in its administration for neuropathic pain conditions.

\section{Procedural interventions}

Procedural interventions have been reported, and range from less-invasive techniques such as injection of botulinum toxin and sympathetic blockade to highly invasive procedures such as implantation of spinal cord stimulators. The reported cases are small in number, and these are primarily attempted in patients who were assessed as refractory to pharmacologic management.

Therapy with botulinum toxin type A with successful reduction in the episode and intensity of pain has been reported in one case report. ${ }^{74}$ The mechanism of action of botulinum toxin is through inhibition of acetylcholine from 
the cholinergic nerve terminals, but this does not explain the analgesic activities. Proposed analgesic mechanism is through an inhibition of peripheral and central sensitization by blocking the release of proinflammatory neurotransmitters such as substance $\mathrm{P}$ and glutamate. Botulinum toxin has been trialed for diabetic neuropathic pain and trigeminal neuralgia, and its application in EM has been proposed as a potentially less invasive treatment modality.

There are several case reports of EM of lower extremity pain being treated with procedures targeting the thoracic and lumbar sympathetic system in adults and children. One hypothesis of pain in EM is that it is a result of tissue hypoxemia from abnormal microvascular blood flow, and that the pain may be sympathetically mediated; sympathetic blockade may ultimately increase tissue perfusion and relieve ischemic pain. These procedures are usually conducted in the setting of refractory cases, as there are increased risks of complications (unintentional tissue damage, neuritis, motor deficits). The types of interventions include epidural or sympathetic blocks, sympathectomy (chemical, radiofrequency ablation, surgical), and spinal cord stimulators. Epidural infusions of bupivacaine were reported to result in complete remission in two patients. ${ }^{75,76}$ For sympathetic blockade, different methods and agents (local anesthetics, phenol, corticosteroids, clonidine) have been used. Various methods of enhancing the success of blockade are recommended to increase the precision of the target, including fluoroscopy and computed tomography-guided procedures. ${ }^{77-80}$

There are three case reports of spinal cord stimulator implantation for refractory pain. ${ }^{81-83}$ This was conducted in patients with refractory pain and was considered after successful trial by placing percutaneous spinal cord stimulator electrodes in the epidural space. Several theories have been proposed for this: spinal cord stimulation selectively blocks a subgroup of $\mathrm{C}$ or $\mathrm{A} \delta$ fibers, and neuromodulation via activation of the $A \beta$ fibers by gate control theory. Neurosurgical intervention was conducted in three pediatric patients ${ }^{112}$ who underwent stereotactic destruction of ventral posterolateral nucleus and centromedian nucleus. One patient demonstrated resolution from secondary EM with correction of multiple spinal cord malformations. ${ }^{84}$

\section{Nonpharmacologic intervention}

Evidence-based treatment of chronic pain conditions includes a multidisciplinary approach. This is also recommended for pain related to EM. There are limited studies that have evaluated the impact of nonpharmacologic interventions.
The efficacy of an intensive pain rehabilitation program has been evaluated in eight adults with EM. There was a significant improvement in physical and emotional functioning, with a reduction in depressive symptoms and negative pain-related cognitions. ${ }^{85}$ This suggested a potential role of a multidisciplinary rehabilitation approach focused on increasing functioning in various domains that may be significantly reduced in patients with severe pain experiences.

With regard to other nonpharmacologic interventions, there are limited studies. Single case reports have been published on pain reduction for patients through hypnosis, biofeedback treatment, and massage therapy. ${ }^{86-88}$

\section{Discussion}

There has been substantial advancement in the understanding of the pathophysiology underlying EM. However, research findings have yet to translate to clinical practice. With no consensus or established guidelines for treatment, the approach for this difficult-to-treat condition has been mostly by trial and error. Yet, there are aspects in the evaluation and management of EM that can be incorporated in order to provide a more systematic approach for patients.

First, establishing the diagnosis of EM may require further testing to rule out other differential diagnoses. There are no formal diagnostic criteria, and the diagnosis of EM is usually based on the hallmark symptoms of erythematous, warm, painful limbs precipitated by heat and relieved by cold conditions. Other conditions resembling clinical presentation need to be excluded. Second, screening may need to be undertaken for associated disease processes. Third, investigation of a potential genetic cause for EM may guide the choice of pharmacologic therapy. Fourth, a multidisciplinary approach should be adopted for treatment of pain. This should incorporate a detailed assessment of the impact of pain on self-care, physical activities, job and school attendance, psychosocial functioning, sleep, and participation in normative roles. Finally, it is important to engage the patient and family in physical and psychological therapies with the goal of optimizing function, in addition to pain reduction.

The diagnosis of EM is based on evaluation of the symptom profile and exclusion of other differential diagnosis. This may include myeloproliferative disorders, connective tissue diseases, malignancy, and peripheral neuropathy. Diagnoses that present with similar features include complex regional pain syndrome and Fabry disease. ${ }^{89}$ There are some differences in patients with complex regional pain syndrome. In the majority of cases, there is unilateral limb involvement and cold stimuli will usually worsen the pain. As for Fabry 
disease, it is an X-linked lysosomal storage disorder due to $\alpha$-galactosidase deficiency secondary to GLA gene mutation. Pain is an early symptom localized to the hands and feet with hyperalgesia or allodynia. This may be triggered by extremes of temperature. Laboratory testing for $\alpha$-galactosidase can be conducted to rule out Fabry disease. There are also reports of EM symptoms secondary to drugs, and cessation of the offending agent has been reported to be associated with resolution of symptoms (Table 1). For secondary EM, documented associations include myeloproliferative diseases such as polycythemia vera, idiopathic thrombocytosis, or other conditions such as diabetes mellitus (Table 2). This has clinical significance as treatment of the underlying disease process may reduce EM symptomatology. Targeted specific therapy based on the pathophysiology of EM may be more effective, for example, aspirin may be more effective in patients with EM and polycythemia vera.

With the diagnosis of inherited EM, the exact genetic mutation may provide some guidance regarding medication management. Inherited EM is usually resistant to most pharmacologic therapies, and the genetic differences in the affected sodium channel may account for therapeutic inefficiency in certain individuals. ${ }^{50}$ For this group of patients, detailed family history should be undertaken with the guidance of a genetic counselor to assess for potential inheritance. Family members should receive genetic counseling and evaluation for treatment.

Most patients with EM require multimodal pharmacotherapy, specifically targeting both pathophysiology and neuropathic pain. As there are variable responses to a wide range of therapeutic options and limited high-quality data, no universally effective treatment regimen has been established. This can result in a frustrating process of trial and error for the patient and treating team, while the patient suffers from severe pain episodes. Quite often, a combination of modalities may be required to provide effective management. A

Table I Differential diagnoses of erythromelalgia

Hereditary sensory and autonomic neuropathies/genetic diseases
Fabry disease
Olmsted syndrome
Neurologic disorders
Complex regional pain syndrome
Peripheral neuropathies (diabetes mellitus, nutritional deficiency)
Vascular
Vessel thrombosis
Infectious diseases
Cellulitis

Table 2 Disorders reported to be associated with erythromelalgia

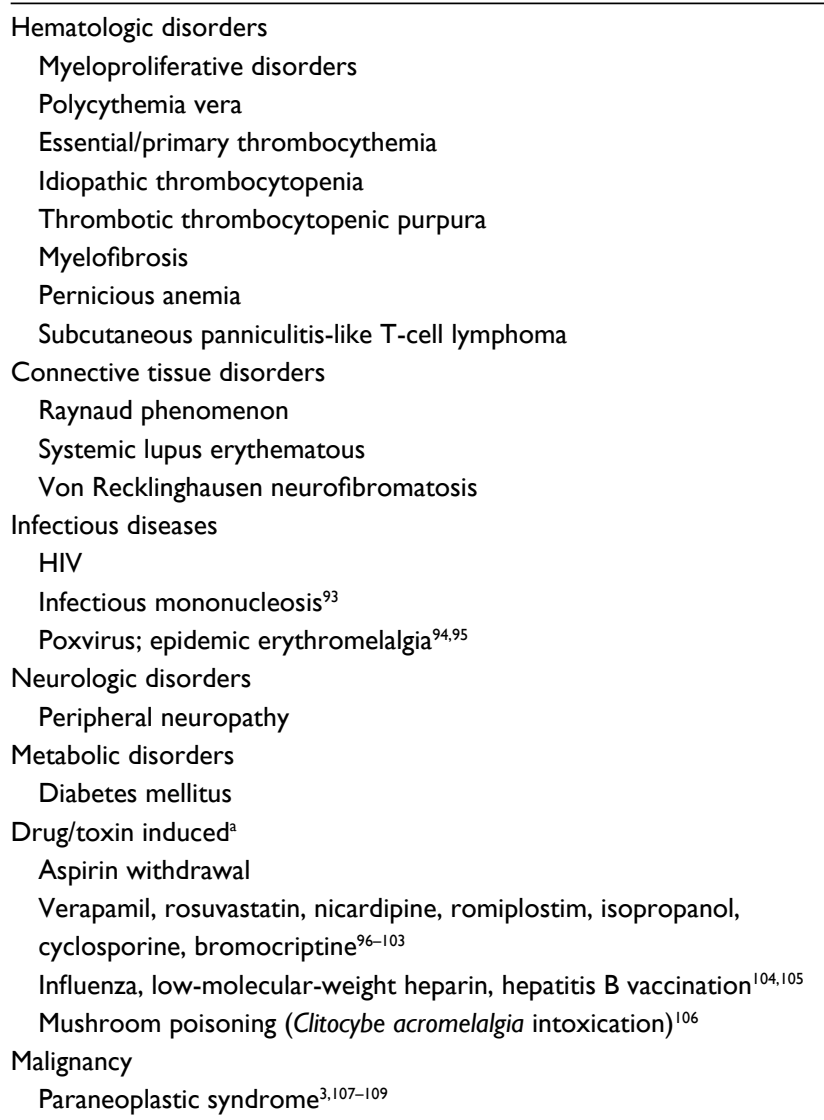

Note: a Medications that have been reported to account for symptoms have also been identified as treatment.

common starting point would be to trial agents with least risk and most likely efficacy. For the patient diagnosed with inherited EM, knowledge of underlying genetic mutation may allow specific sodium channel blockers as the first medication trial. If unknown, oxcarbazepine or carbamezepine may be initiated and titrated to maximum dose. If ineffective, lidocaine infusion may be considered, followed by mexiletine if it shows efficacy in pain reduction. Adjunct treatment with antidepressants and gabapentinoids may be added with the goal of treating central causes of pain. Other agents such as antihistamines that may have vascular mechanisms may be added to the regimen. A strategic approach would be to discontinue agents that do not have any efficacy, in order to avoid polypharmacy and the potential adverse effects of medication interactions. Finally, physical and occupational therapy, rehabilitation, and pain psychology treatment would also be recommended. Other functional domains such as sleep disturbances should also be addressed, including the use of pharmacotherapy and cognitive behavioral therapy, due to the bidirectional relationship between sleep and pain. 


\section{Limitations}

There are several limitations of this review. First, there are no RCTs that evaluate for the efficacy of a single commercially available pharmacologic agent, or compared nonpharmacologic approaches. Second, the available studies have small sample sizes and are primarily composed of retrospective reviews and case reports. Third, there are limited reports on long-term outcomes. Finally, it is also unclear whether there are differences between pediatric and adult populations with regard to the etiology of EM and treatment outcomes. All these factors highlight the challenges of testing the impact of pharmacologic and/or nonpharmacologic treatment. There are opportunities for research in this area, including through collaborative consortiums to collectively gather data on this rare condition.

\section{Conclusion}

EM is a challenging condition to treat due to our lack of understanding of the pathomechanisms of the disease process. To date, treatment is a stepwise trial and error approach, and no single therapy has been found to be effective for any patient. In addition to partial responses, there is varying response to pharmacotherapy and significant variability within this clinical population to treatment. This may be due to the etiology of EM or the natural course of disease process. Therefore, incorporating a multidisciplinary approach to address pain and its significant negative impact across many domains of functioning may target the goals of improving function, in addition to pain reduction in this difficult-totreat population. Numerous investigations have extended our understanding of the underlying mechanisms of EM. Most recently, Farrar et al identified acute axonal depolarization and ischemia that occurs with heat, providing an insight into underlying pathophysiology of EM. ${ }^{90}$ Genetic evidence also supports investigative efforts to develop Nav1.7 blockers as a novel therapeutic strategy. These are undergoing clinical trials. ${ }^{2,91}$ Finally, advancement in electrophysiology, molecular modeling, thermodynamic analysis, and functional analyses profiling is improving our understanding of the molecular mechanisms underlying pain in EM. ${ }^{51}$ These studies are much needed to address the pain and significant comorbidity in patients with EM.

\section{Disclosure}

The authors report no conflicts of interest in this work.

\section{References}

1. Mørk C, Kvernebo K. Erythromelalgia-A Mysterious Condition? Arch Dermatol. 2000;136(3):406-409.
2. Tang Z, Chen Z, Tang B, Jiang H. Primary erythromelalgia: a review. Orphanet J Rare Dis. 2015;10(1):127.

3. Alhadad A, Wollmer P, Svensson A, Eriksson KF. Erythromelalgia: Incidence and clinical experience in a single centre in Sweden. Vasa. 2012;41(1):43-48.

4. Reed KB, Davis MDP. Incidence of erythromelalgia: a populationbased study in Olmsted County, Minnesota. Journal of the European Academy of Dermatology and Venereology. 2009;23(1):13-15.

5. Kalgaard OM, Seem E, Kvernebo K. Erythromelalgia: a clinical study of 87 cases. J Intern Med. 1997;242(3):191-197.

6. Heidrich H. Functional vascular diseases: Raynaud's syndrome, acrocyanosis and erythromelalgia. Vasa. 2010;39(1):33-41.

7. Parker LK, Ponte C, Howell KJ, Ong VH, Denton CP, Schreiber BE. Clinical features and management of erythromelalgia: long-term follow-up of 46 cases. Clinical and Experimental Rheumatology. 2017;35:80-84.

8. Johnson E, Iyer P, Eanes A, Zolnoun D. Erythema and Burning Pain in the Vulva: A Possible Phenotype of Erythromelalgia. Case Rep Med. 2011;2011(3): Article ID 374167.

9. Messeguer F, Agusti-Mejias A, Vilata Corell JJ, Requena C. Auricular erythromelalgia: report of a rare case. Dermatol Online J. 2013; 19:16.

10. Khan S, Affleck A. Periodic facial erythema in a patient with postural orthostatic tachycardia syndrome. Clin Exp Dermatol. 2018;43(1): $100-102$.

11. Dib-Hajj SD, Rush AM, Cummins TR, et al. Gain-of-function mutation in Nav1.7 in familial erythromelalgia induces bursting of sensory neurons. Brain. 2005;128(8):1847-1854.

12. Davis MDP. Immersion foot associated with the overuse of ice, cold water, and fans: a distinctive clinical presentation complicating the syndrome of erythromelalgia. J Am Acad Dermatol. 2013;69(1): 169-171.

13. Kirby RL. Erythromelalgia-not so benign. Arch Phys Med Rehabil. 1987;68:389.

14. Cook-Norris RH, Tollefson MM, Cruz-Inigo AE, Sandroni P, Davis MD, Davis DM. Pediatric erythromelalgia: a retrospective review of 32 cases evaluated at Mayo Clinic over a 37-year period. J Am Acad Dermatol. 2012;66(3):416-423.

15. Davis MDP, O'Fallon WM, Rogers RS, Rooke TW. Natural history of erythromelalgia - Presentation and outcome in 168 patients. Archives of Dermatology. 2000;136:330-336.

16. Friberg D, Chen T, Tarr G, van Rij A. Erythromelalgia? A clinical study of people who experience red, hot, painful feet in the community. Int J Vasc Med. 2013;2013(2): Article ID 864961.

17. Dib-Hajj SD, Yang Y, Waxman SG. Genetics and molecular pathophysiology of $\mathrm{Na}(\mathrm{v}) 1.7$-related pain syndromes. Adv Genet. 2008;63:85-110

18. Mcdonnell A, Schulman B, Ali Z, et al. Inherited erythromelalgia due to mutations in SCN9A: natural history, clinical phenotype and somatosensory profile. Brain. 2016;139(4):1052-1065.

19. Dabby R. Pain disorders and erythromelalgia caused by voltagegated sodium channel mutations. Curr Neurol Neurosci Rep. 2012;12(1):76-83.

20. Tanaka BS, Nguyen PT, Zhou EY, et al. Gain-of-function mutation of a voltage-gated sodium channel $\mathrm{Na}_{\mathrm{V}} 1.7$ associated with peripheral pain and impaired limb development. Journal of Biological Chemistry. 2017;292(22):9262-9272.

21. Ørstavik K, Mørk C, Kvernebo K, Jørum E. Pain in primary erythromelalgia - a neuropathic component? Pain. 2004;110(3):531-538.

22. Genebriera J, Michaels JD, Sandroni P, Davis MDP. Results of computer-assisted sensory evaluation in 41 patients with erythromelalgia. Clin Exp Dermatol. 2012;37(4):350-354.

23. Davis MDP, Weenig RH, Genebriera J, Wendelschafer-Crabb G, Kennedy WR, Sandroni P. Histopathologic findings in primary erythromelalgia are nonspecific: Special studies show a decrease in small nerve fiber density. J Am Acad Dermatol. 2006;55(3):519-522.

24. Oaklander A. Erythromelalgia: Small-Fiber Neuropathy by Any Other Name? Pediatrics. 2005;116(1); :293-294. 
25. Davis MD, Sandroni P, Rooke TW, Low PA. Erythromelalgia: vasculopathy, neuropathy, or both? A prospective study of vascular and neurophysiologic studies in erythromelalgia. Arch Dermatol. 2003;139: 1337-1343.

26. Davis MDP, Sandroni P, Harper CM, et al. Neurophysiologic and vascular studies in erythromelalgia: A retrospective analysis. Journal of Investigative Dermatology. 1998;110:590.

27. Tham SW, Li L, Effraim P, Waxman S. Between fire and ice: refractory hypothermia and warmth-induced pain in inherited erythromelalgia BMJ Case Rep. 2017;2017:bcr-2017-219486.

28. Michiels JJ. Aspirin responsive erythromelalgia in JAK2-thrombocythemia and incurable inherited erythrothermalgia in neuropathic Nav1.7 sodium channelopathy: from Mitchell 1878 to Michiels 2017. Expert Opin Orphan Drugs. 2017;5(2):111-129.

29. Willoughby S, Pearson TC. The use of aspirin in polycythaemia vera and primary thrombocythaemia. Blood Rev. 1998;12(1):12-22.

30. Ruggeri M, Castaman G, Rodeghiero F. Is ticlopidine a safe alternative to aspirin for management of myeloproliferative disorders? Haematologica. 1993;78:18-21.

31. Calderone DC, Finzi E. Treatment of primary erythromelalgia with piroxicam. J Am Acad Dermatol. 1991;24(1):145-146.

32. Chan TLH, Becker WJ, Jog M. Indomethacin-Responsive Idiopathic Red Ear Syndrome: Case Report and Pathophysiology. Headache. 2018;58(2):306-308.

33. Kalgaard OM, Clausen OP, Mellbye OJ, Hovig T, Kvernebo K. Nonspecific Capillary Proliferation and Vasculopathy Indicate Skin Hypoxia in Erythromelalgia. Arch Dermatol. 2011;147(3):309-314.

34. Kalgaard O, Mørk C, Kvernebo K. Prostacyclin Reduces Symptoms and Sympathetic Dysfunction in Erythromelalgia in a Doubleblind Randomized Pilot Study. Acta Derm Venereol. 2003;83(6): 442-444.

35. Mørk C, Göran Salerud E, Asker CL, Kvernebo K. The Prostaglandin E1 Analog Misoprostol Reduces Symptoms and Microvascular Arteriovenous Shunting in Erythromelalgia-A Double-Blind, Crossover Placebo-Compared Study. J Invest Dermatol. 2004;122(3):587-593.

36. Goldberg YP, Price N, Namdari R, et al. Treatment of Nav1.7-mediated pain in inherited erythromelalgia using a novel sodium channel blocker. Pain. 2012;153(1):80-85.

37. Cao L, Mcdonnell A, Nitzsche A, et al. Pharmacological reversal of a pain phenotype in iPSC-derived sensory neurons and patients with inherited erythromelalgia. Sci Transl Med. 2016;8(335):ra356.

38. Elgueta F, de La Cuadra-Fontaine JC, Clede L, Fierro C, Valderrama A. Erythromelagia: A Rare and Hard-to-Treat Condition: A 9-Year-Old Boy Responsive to Intravenous Lidocaine and Oral Mexilitene. Pain Medicine. 2013;14(2):311-312.

39. Kuhnert SM, Phillips WJ, Davis MD. Lidocaine and mexiletine therapy for erythromelalgia. Arch Dermatol. 1999;135(12):1447-1449.

40. Jakob A, Creutzfeldt R, Staszewski O, Winterpacht A, Berner R, Hufnagel M. Primary Erythromelalgia in a 12-Year-Old Boy: Positive Response to Sodium Channel Blockers Despite Negative SCN9A Mutations. Klinische Pädiatrie. 2012;224(05):309-312.

41. Sheets PL, Jackson JO, Waxman SG, Dib-Hajj SD, Cummins TR. A $\mathrm{Na}_{\mathrm{v}} 1.7$ channel mutation associated with hereditary erythromelalgia contributes to neuronal hyperexcitability and displays reduced lidocaine sensitivity. J Physiol. 2007;581(Pt 3):1019-1031.

42. Helås T, Sagafos D, Kleggetveit IP, et al. Pain thresholds, supra -threshold pain and lidocaine sensitivity in patients with erythromelalgia, including the I848Tmutation in $\mathrm{Na}_{\mathrm{v}}$ 1.7. European Journal of Pain. 2017;21(8):1316-1325

43. Paticoff J, Valovska A, Nedeljkovic SS, Oaklander AL. Defining a Treatable Cause of Erythromelalgia: Acute Adolescent Autoimmune SmallFiber Axonopathy. Anesthesia \& Analgesia. 2007;104(2):438-441.

44. Iqbal J, Bhat M, Charoo B, Syed W, Sheikh M, Bhat I. Experience with oral mexiletine in primary erythromelalgia in children. Ann Saudi Med. 2009;29(4):316-318.

45. Kuhnert SM, Phillips WJ, Davis MDP. Lidocaine and mexiletine therapy for erythromelalgia. Arch Dermatol. 1999;135(12):1447-1449.
46. Nathan A, Rose JB, Guite JW, Hehir D, Milovcich K. Primary Erythromelalgia in a Child Responding to Intravenous Lidocaine and Oral Mexiletine Treatment. Pediatrics. 2005;115(4):e504-e507.

47. Jang H-S, Jung D, Kim S, et al. A case of primary erythromelalgia improved by mexiletine. Br J Dermatol. 2004;151(3):708-710.

48. Vivas AC, Escandon J, Kirsner RS. Refractory erythromelalgia of the ears: response to mexiletine. Am J Otolaryngol. 2011;32(2):168-170.

49. Natkunarajah J, Atherton D, Elmslie F, Mansour S, Mortimer P. Treatment with carbamazepine and gabapentin of a patient with primary erythermalgia (erythromelalgia) identified to have a mutation in the SCN9A gene, encoding a voltage-gated sodium channel. Clin Exp Dermatol. 2009;34(8):e640-e642.

50. Fischer TZ, Gilmore ES, Estacion M, et al. A novel Na 1.7 mutation producing carbamazepine-responsive erythromelalgia. Ann Neurol. 2009;65(6):733-741.

51. Geha P, Yang Y, Estacion M, et al. Pharmacotherapy for pain in a family with inherited erythromelalgia guided by genomic analysis and functional profiling. JAMA Neurol. 2016;73(6):659-667.

52. Cohen JS. High-dose oral magnesium treatment of chronic, intractable erythromelalgia. Ann Pharmacother. 2002;36(2):255-260.

53. Rudikoff D, Jaffe IA. Erythromelalgia: Response to serotonin reuptake inhibitors. J Am Acad Dermatol. 1997;37(2):281-283.

54. Firmin D, Roguedas AM, Greco M, et al. Treatment of familial erythromelalgia with venlafaxine. Journal of the European Academy of Dermatology and Venereology. 2007;21(6):836-837.

55. Cohen JS. Erythromelalgia: New theories and new therapies. $J A m$ Acad Dermatol. 2000;43(5):841-847.

56. Mcgraw T, Kosek P. Erythromelalgia pain managed with gabapentin Anesthesiology. 1997;86(4):988-990.

57. Kakizaki A, Fujimura T, Kambayashi Y, Watabe A, Aiba S. Successful treatment of adult-onset erythromelalgia with steroid pulse and pregabalin. Case Rep Dermatol. 2012;4(3):242-246.

58. Kalava K, Roberts C, Adair JD, Raman V. Response of primary erythromelalgia to pregabalin therapy. Journal of Clinical Rheumatology. 2013;19(5):284-285

59. Sakakibara R, Fukutake T, Kita K, Hattori T. Treatment of primary erythromelalgia with cyproheptadine. J Auton Nerv Syst. 1996;58(1-2):121-122.

60. Al-Minshawy SM, El-Mazary A-AM. An Egyptian child with erythromelalgia responding to a new line of treatment: a case report and review of the literature. J Med Case Rep. 2014;8(1):69.

61. Chan MKH, Tucker AT, Madden S, Golding CE, Atherton DJ, Dillon MJ. Erythromelalgia: an endothelial disorder responsive to sodium nitroprusside. Arch Dis Child. 2002;87(3):229-230.

62. Zsoylu S, Coskun T. Sodium nitroprusside treatment in erythromelalgia. Eur J Pediatr. 1984;141(3):185-187.

63. Poterucha TJ, Murphy SL, Davis MDP, et al. Topical amitriptyline combined with ketamine for the treatment of erythromelalgia: a retrospective study of 36 patients at mayo clinic. Journal of Drugs in Dermatology. 2013;12:308-310.

64. Davis MDP, Sandroni P. Lidocaine Patch for Pain of Erythromelalgia. Arch Dermatol. 2002;138(1):17-19.

65. Davis MD, Sandroni P. Lidocaine patch for pain of erythromelalgia: follow-up of 34 patients. Arch Dermatol. 2005;141:1320-1321.

66. Davis MDP, Morr CS, Warndahl RA, Sandroni P. Topically Applied Midodrine, $0.2 \%$, an $\alpha_{1}$-Agonist, for the Treatment of Erythromelalgia. JAMA Dermatol. 2015;151(9):1025-1026.

67. Muhiddin KA, Gallen IW, Harries S, Pearce VR. The use of capsaicin cream in a case of erythromelalgia. Postgrad Med J. 1994;70(829): 841-843.

68. Pagani-Estévez GL, Sandroni P, Davis MD, Watson JC. Erythromelalgia: identification of a corticosteroid-responsive subset. $J$ Am Acad Dermatol. 2017;76(3):506-511.

69. Pfund Z, Stankovics J, Decsi T, Illes Z. Childhood steroid-responsive acute erythromelalgia with axonal neuropathy of large myelinated fibers: A dysimmune neuropathy? Neuromuscular Disorders. 2009;19(1):49-52. 
70. Jackson AL, Oates JA. A Patient With Adult Erythermalgia: Evidence Suggesting an Autoimmune Etiology. Am J Med Sci. 2008;335(4):320-322.

71. Moody S, Pacheco S, Butler IJ, Koenig MK. Secondary Erythromelalgia Successfully Treated With Intravenous Immunoglobulin. J Child Neurol. 2012;27(7):922-923.

72. Nakajima N, Ueda M. Nicotinic acid treatment for Paralepistopsis acromelalga intoxication: assessment using magnetic resonance imaging. Clin Toxicol. 2016;54(7):597-600.

73. Parker LK, Ponte C, Howell KJ, Ong VH, Denton CP, Schreiber BE. Clinical features and management of erythromelalgia: long term follow-up of 46 cases. Clin Exp Rheumatol. 2017;35:80-84.

74. Costa A, Meireles J, Festas MJ, Gomes A, Abreu P. Therapeutic success with local botulinum toxin in erythromelalgia. Pain Physician. 2014;17:E658-E660.

75. D'Angelo R, Cohen IT, Brandom BW. Continuous epidural infusion of bupivacaine and fentanyl for erythromelalgia in an adolescent. Anesth Analg. 1992;74:142-144.

76. Stricker LJ, Green CR. Resolution of Refractory Symptoms of Secondary Erythermalgia With Intermittent Epidural Bupivacaine. Reg Anesth Pain Med. 2001;26(5):488-490.

77. Kundu A, Rafiq M, Warren P, Tobias J. Erythromelalgia in the pediatric patient: role of computed-tomography-guided lumbar sympathetic blockade. Journal of Pain Research. 2016;9:837-845.

78. Cerci FB, Kapural L, Yosipovitch G. Intractable erythromelalgia of the lower extremities successfully treated with lumbar sympathetic block. J Am Acad Dermatol. 2013;69(5):e270-e272.

79. Bang YJ, Yeo JS, Kim SO, Park YH. Sympathetic Block for Treating Primary Erythromelalgia. Korean J Pain. 2010;23(1):55-59.

80. Shiga T, Sakamoto A, Koizumi K, Ogawa R. Endoscopic Thoracic Sympathectomy for Primary Erythromelalgia in the Upper Extremities. Anesthesia \& Analgesia. 1999;88(4):865-866.

81. Matzke LL, Lamer TJ, Gazelka HM. Spinal cord stimulation for treatment of neuropathic pain associated with erythromelalgia. Reg Anesth Pain Med. 2016;41(5):619-620.

82. Graziotti PJ, Goucke CR. Control of intractable pain in erythromelalgia by using spinal cord stimulation. J Pain Symptom Manage. 1993;8(7):502-504.

83. Patel N, Chen E, Cucchiaro G. The complexity of pain management in patients with erythromelalgia. A A Case Rep. 2015;5(9):151-153.

84. Mosel DD, Rosler D. Erythromelalgia presenting after neurosurgical intervention in a patient with multiple malformations of the spinal cord. J Am Acad Dermatol. 2011;65(4):e120-e122.

85. Durosaro O, Davis MD, Hooten WM, Kerkvliet JL. Intervention for erythromelalgia, a chronic pain syndrome: comprehensive pain rehabilitation center, Mayo Clinic. Arch Dermatol. 2008;144:1578-1583.

86. Putt AM. Erythromelalgia-a case for biofeedback. Nurs Clin North Am. 1978;13:625-630

87. Dicks K, Rizek P. Massage therapy techniques as pain management for erythromelalgia: a case report. Int JTher Massage Bodywork. 2010;3:5-9.

88. Chakravarty K, Pharoah PD, Scott DG, Barker S. Erythromelalgia--the role of hypnotherapy. Postgrad Med J. 1992;68(795):44-46.

89. Chen Z, Ye W, Jiao SJ, et al. Diagnosis of a pedigree with Fabry disease mimicking erythromelalgia: the utility of next-generation sequencing in a precision medicine perspective. International Journal of Clinical and Experimental Medicine. 2016;9:16923-16926.

Journal of Pain Research

\section{Publish your work in this journal}

The Journal of Pain Research is an international, peer reviewed, open access, online journal that welcomes laboratory and clinical findings in the fields of pain research and the prevention and management of pain. Original research, reviews, symposium reports, hypothesis formation and commentaries are all considered for publication.
90. Farrar MA, Lee MJ, Howells J, Andrews PI, Lin CS. Burning pain: axonal dysfunction in erythromelalgia. Pain. 2017;158:900-911.

91. Dib-Hajj SD, Geha P, Waxman SG. Sodium channels in pain disorders: pathophysiology and prospects for treatment. Pain. 2017;158:S97-S107.

92. Eaton M, Murphy S. Erythromelalgia misdiagnosed as cellulitis. Cutis. 2005;75:37-40.

93. Clayton C, Faden H. Erythromelalgia in a twenty-year-old with infectious mononucleosis. Pediatr Infect Dis J. 1993;12:101-102.

94. Gu Y, Chen F, Liu T, et al. Early detection of an epidemic erythromelalgia outbreak using Baidu search data. Sci Rep. 2015;5(1):12649.

95. Zheng ZM, Zhang JH, Hu JM, Liu SF, Zhu WP. Poxviruses isolated from epidemic erythromelalgia In china. The Lancet. 1988; 331(8580):296.

96. Rajabally YA, Mortimer NJ. Acute neuropathy and erythromelalgia following topical exposure to isopropanol. Vet Hum Toxicol. 2004;46:24-25.

97. Cimolai N, Cimolai TL. Erythromelalgia accompanying rosuvastatinassociated myopathy. J Dermatol Case Rep. 2009;3(1):1-3.

98. Drenth JP. Erythromelalgia induced by nicardipine. BMJ. 1989;298(6687): 1582-1582.

99. Thami GP, Bhalla M. Drug points: erythromelalgia induced by possible calcium channel blockade by ciclosporin. BMJ. 2003;326(7395): 910.

100. Ali A, Rothner A. Erythromelalgia secondary to verapamil. Headache. 2017;57:168-169.

101. Dulon M. Romiplostim-induced erythromelalgia in a patient with idiopathic thrombocytopenic purpura. British Journal of Dermatology. 2009;161:482-482.

102. Nanayakkara PW, van der Veldt AA, Simsek S, Smulders YM, Rauwerda JA. Verapamil-induced erythermalgia. Neth J Med. 2007;65(9): 349-351.

103. Drenth JP, Michiels JJ, van Joost T, Vuzevski VD. Verapamil-induced secondary erythermalgia. Br J Dermatol. 1992;127(3):292-294.

104. Confino I, Passwell JH, Padeh S. Erythromelalgia following influenza vaccine in a child.

105. Rabaud C, Barbaud A, Trechot P. First case of erythermalgia related to hepatitis B vaccination. J Rheumatol. 1999;26(1):233-234.

106. Saviuc P, Danel V. New syndromes in mushroom poisoning. Toxicol Rev. 2006;25(3):199-209.

107. Kurzrock R, Cohen PR. Paraneoplastic erythromelalgia. Clin Dermatol. 1993;11(1):73-82.

108. Han JH, Lee JB, Kim SJ, Lee SC, Won YH, Yun SJ. Paraneoplastic erythromelalgia associated with breast carcinoma. Int $J$ Dermatol. 2012;51(7):878-880.

109. Protopsaltis I, Drossou A, Katsantonis I, et al. Breast cancer presenting as paraneoplastic erythroderma: an extremely rare case. Case Rep Med. 2014;2014: Article ID 351065.

110. Pepper H. Primary erythermalgia: report of a patient treated with methysergide maleate. JAMA. 1968;203(12):1066-1067.

111. Cohen JS. Erythromelalgia: new theories and new therapies. $J \mathrm{Am}$ Acad Dermatol. 2000;43(5 Pt 1):841-847.

112. Kandel EI. Stereotactic surgery of erythromelalgia. Stereotact Funct Neurosurg. 1990;54-55:96-100.

The manuscript management system is completely online and includes a very quick and fair peer-review system, which is all easy to use. Visit http://www.dovepress.com/testimonials.php to read real quotes from published authors. 